12. Zhu S, Wang Z, Heshka S, Heo M, Faith MS, Heymsfield SB. Waist circumference and obesity-associated risk factors among whites in the third National Health and Nutrition Examination Survey: clinical action thresholds. Am 7 Clin Nutr 2002;76:743-9.

13. Ohlson LO, Larsson B, Svardsudd K, Welin L, Eriksson H, Wilhelmsen L, et al. The influence of body fat distribution on the incidence of diabetes mellitus: 13.5 years of follow-up of the participants in the study of men born in 1913. Diabetes 1985;34:1055-8.

14. Lemieux I, Pascot A, Couillard C, Lamarche B, Tchernof A, Almeras N, et al. Hypertriglyceridemic waist: a marker of the atherogenic metabolic triad (hyperinsulinemia; hyperapolipoprotein B; small, dense LDL) in men? Circu- lation 2000;102:179-84

15. National Institutes of Health. The practical guide: identification, evaluation and treatment of overweight and obesity in adults. Bethesda (MD): The Institutes; 2000.

Correspondence to: Dr. Simone Lemieux, 2440 Hochelaga Blvd., Institute of Nutraceuticals and Functional Foods, Laval University, Québec QC G1K 7P4; fax 418 656-5877;

simone.lemieux@aln.ulaval.ca

\title{
Transparency in drug regulation: Mirage or oasis?
}

\section{Joel Lexchin, Barbara Mintzes}

I n Canada, the information used to approve new drugs is deemed commercially sensitive and hence confidential under the Access to Information Act, ${ }^{1}$ and the Therapeutic Products Directorate (TPD) will not release such information without the manufacturer's approval. As a consequence, safety and efficacy information contained in unpublished trials submitted to the TPD is generally unavailable to researchers, physicians and patients, a situation that can potentially lead to the inappropriate prescribing and use of medications.

The standard argument for the legal protection of these data is that their disclosure would compromise the economic interests of drug manufacturers. This rationale is difficult to credit in view of experience in other jurisdictions. The US Food and Drug Administration (FDA) discloses research information from preclinical and clinical trials that is considered proprietary in Canada, without apparent negative effects on companies' profitability or willingness to operate in the US market. For example, extensive information on rosuvastatin is available on the FDA's Web site. ${ }^{2}$

On the other hand, nondisclosure has serious disadvantages for the TPD, health professionals and the Canadian public. If scientific data submitted to regulatory agencies are never disclosed or allowed to enter normal peer-review channels, neither these data nor TPD reviewers' evaluations can become subject to scrutiny by independent scientists. The scientific atmosphere of the agency may be stifled and the professional growth of its staff severely inhibited. ${ }^{3}$ Deprived of any independent access to information, health professionals and the public must accept the TPD's judgement about the safety and effectiveness of products.

The level of secrecy in the TPD has been criticized on a number of occasions, including in a 2000 report by the ad hoc Committee on the Drug Review Process of Health Canada's own Science Advisory Board. The report stated:
$[\mathrm{I}] \mathrm{n}$ our view and that of many stakeholders, the current drug review process is unnecessarily opaque. Health Canada persists in maintaining a level of confidentiality that is inconsistent with public expectation and contributes to a public cynicism about the integrity of the process. ${ }^{4}$

To remedy this situation the Committee recommended

that HPB [Health Protection Branch, now Health Products and Food Branch] should set new standards of access to information at all stages of the drug review process, enhancing transparency and public confidence. ${ }^{4}$

In 2004 a report by the House of Commons Standing Committee on Health supported the development of mechanisms to enable greater public disclosure of information about clinical trials. ${ }^{5}$

In response to these calls for greater transparency, the TPD announced in 2004 that when new drugs and devices are approved it would publish a document entitled the Summary Basis of Decision (SBD). The SBD would outline the scientific and benefit/risk-based reasons for the TPD's decision to grant market authorization for a product. ${ }^{6}$

The key part of the SBD of importance to prescribers and consumers is the clinical information on drug effectiveness and safety. Is enough information provided to allow for the safe and rational use of new medications or extension of indications for previously approved drugs?

To evaluate the adequacy of information in the SBDs, we examine 3 recent cases in which unpublished data submitted to drug regulators contained important clinical information that was either unavailable or misrepresented within the published literature. We ask whether the same discoveries would have been possible using Health Canada's SBDs. We based our assessment on 2 pilot SBDs published to date, one for rosuvastatin, ${ }^{7}$ a cholesterol-lowering medication, the second for agalsidase beta, ${ }^{8}$ an enzyme replacement for use in Fabry's disease. 


\section{1: Celecoxib}

Celecoxib is a COX-2 inhibitor whose purported benefit over older anti-inflammatory agents (NSAIDs) is that it causes fewer serious gastrointestinal side effects. A study published in the Fournal of the American Medical Association (FAMA) in 2000 appeared to confirm this assertion. ${ }^{9}$

However, material on the FDA Web site revealed discrepancies between the data as published in $\mathcal{F} A M A$ and that submitted to the FDA. ${ }^{10,11}$ The $\mathcal{F} A M A$ article failed to mention that it was an interim report of the first 6 months of data from 2 trials that lasted 12 and 16 months. Most important, at 65 weeks there was no difference in gastrointestinal adverse effects between celecoxib and traditional NSAID groups. In violation of the methodology outlined in the study protocol, the authors of the $7 A M A$ article made conclusions about subgroups despite the lack of significance in the primary study outcome.

The discrepancies were discovered because the following information was available on the FDA Web site: study protocol, FDA reviewers' comments, and detailed information on trial outcomes. Study protocol and reviewers' comments are not included in the pilot SBDs. Detailed outcomes of individual trials (efficacy results and side effects) are not presented. Therefore, it would have been difficult to determine from an SBD whether the published studies combined the results of more than one clinical trial or if interim trial results were falsely presented as full trial results. In short, most of the problems with the $7 A M A$ study on celecoxib would not have been found using an SBD.

\section{2: Efficacy of antidepressants}

A systematic review published in $B M 7$ in 2003 looked at 42 placebo-controlled studies of 5 selective serotonin reuptake inhibitors. ${ }^{12}$ The Swedish drug regulatory authority granted the authors access to these trials, which were submitted as a basis for marketing approval for treatments for major depression. These studies were then compared with the studies that were eventually published. The authors found 3 types of bias: multiple publication, selective publication and selective reporting. Studies showing significant effects of a drug were published as stand-alone publications more often than studies with nonsignificant results. Many publications ignored the results of intention-to-treat analyses.

Shortfall in information in Health Canada's Summary Basis of Decision documents for rosuvastatin and agalsidase

- Study protocol unavailable

- No information about baseline characteristics of trial participants, number of participants who withdrew and reasons for withdrawal

- No data about primary and secondary efficacy outcomes or fatal and non-fatal serious adverse events by treatment arm
These biases resulted in a more favourable representation of the drugs' effectiveness and safety than the full trial data and could have significantly affected the results of systematic reviews and meta-analyses. They were discovered because the authors had access to all of the information that was submitted to the regulator.

In a second systematic review of antidepressant trials, Kirsch and colleagues analyzed efficacy data submitted to the FDA for approval of 6 widely prescribed antidepressants. ${ }^{13}$ They found overall that $80 \%$ of the response to medication was duplicated in placebo control groups. The mean difference between drug and placebo on the Hamilton Depression Scale was less than 2 points (on a 64-point scale), which the authors judged to be of questionable clinical significance. The difference between drug and placebo was smaller in this analysis than in previous meta-analyses based on published data only.

The information available in Health Canada's pilot SBDs does not include results (efficacy and side effects) for individual trials. Therefore, the difference in bias between the trials submitted to regulatory agencies and the subset of published trials would not have been discovered using an SBD. Additionally, SBDs do not provide the detail needed on trial outcomes for the analysis of the magnitude of drug effect carried out by Kirsch and colleagues.

\section{3: Cardiovascular risks of hormone replacement therapy}

The publication of the results of the Women's Health Initiative study of hormone replacement therapy (HRT) showed that use of estrogen and progestin in healthy postmenopausal women leads to increased cardiovascular risks. ${ }^{14}$ A previous comparison of published and unpublished data submitted to regulatory authorities suggests that these risks could have been uncovered earlier, potentially sparing women adverse health outcomes.

In 1997 Hemminki and colleagues took data on cardiovascular and cancer events from published randomized controlled trials on HRT and showed that the odds ratio for cardiovascular and thromboembolic events for women taking HRT versus those not taking it was 1.64 (95\% confidence interval [C] $0.65-4.21) .{ }^{15}$ In a second analysis, Hemminki added the results from 6 unpublished trials. ${ }^{16}$ The additional data hinted at publication bias, as the relative risk in the unpublished trials was about 4.25 for cardiovascular events, although the overall results did not change significantly. ${ }^{16}$

McPherson and Hemminki concluded that "systematic synthesis of all data from well conducted small clinical (efficacy) trials would have revealed the effect of hormone replacement therapy on cardiovascular risk much earlier, even than 1997 ... [but] many of the studies were unavailable." ${ }^{17}$

To be able to add data from the unpublished trials to the meta-analysis, the authors needed outcome and safety data on each individual unpublished trial. An SBD does not pro- 
vide this information and therefore would not have uncovered the negative risk-benefit ratio for combined HRT in postmenopausal women.

\section{Conclusion}

In each of these examples the information available in the published literature failed to reflect the full body of scientific knowledge about a drug's effects. These problems would not have been discovered using Health Canada's SBDs, which lack detailed information on clinical trial design, methods and outcomes.

A model for the minimum level of reporting already exists in FDA approval packages. Once a drug has been approved in the United States, the FDA posts on its Web site a detailed summary of the information that the company has submitted, including the clinical trial data. Compared with what is already available in the US, this initiative for greater transparency in Canada is grossly inadequate. However, it is not enough for Canada to rely on the US for information: companies may use different datasets when they apply for approval in this country; approved indications may not be the same in Canada and the US; the opinions of TPD drug reviewers about the material that companies submit may differ from those of their American colleagues; and companies may seek approval for drugs that are not on the US market or that were turned down by the FDA. In the latter case, US regulators do not release clinical information.

If the TPD truly wants to expand public access to meaningful information, it needs to go beyond the SBD it is offering. We echo the recommendation of the Science Advisory Committee that "Canada can at least emulate the standards of openness of our nearest and largest trading partner."

A longer version of this paper was previously published by the Canadian Centre for Policy Alternatives.

Dr. Lexchin is an Emergency Physician with the University Health Network, and Associate Professor, School of Health Policy and Management, York University, and is Associate Professor with the Department of Family and Community Medicine, University of Toronto, Toronto, Ont. Dr. Mintzes is a Postdoctoral Fellow in the Department of Pharmacology and Therapeutics, University of British Columbia, Vancouver, BC.
Competing interests: None delared.

Contributors: Joel Lexchin conceived of the idea for this article and wrote the first draft. Barbara Mintzes revised the draft and made important intellectual contributions. Both authors approved the final version.

\section{References}

1. Government of Canada. Access to Information Act. Ottawa; 1985

2. US Food and Drug Administration. Center for Drug Evaluation and Research. Crestor (rosuvastatin calcium) tablets. Available: www.fda.gov /cder/foi/nda/2003/21-366_Crestor.htm (accessed 2004 Oct 30).

3. International Working Group on Transparency and Accountability in Drug Regulation. Statement. Amsterdam: Health Action International; 1996.

4. Science Advisory Board Committee on the Drug Review Process. Report to Health Canada. Ottawa: The Committee; 2000.

5. House of Commons Standing Committee on Health. Opening the medicine cabinet: first report on bealth aspects of prescription drugs. Ottawa: The Committee; 2004.

6. Issue analysis summary: summary basis of decision. Draft 7. Ottawa; Health Canada; 2004.

7. Summary Basis of Decision (SBD) Crestor - AstraZeneca Canada Inc. Ottawa; Health Canada; 2004, Sept 9.

8. Summary Basis of Decision (SBD) Fabrazyme - Genzyme Canada Inc. Ottawa; Health Canada; 2004, Jun 28.

9. Silverstein FE, Faich G, Goldstein JL, Simon LS, Pincus T, Whelton A, et al Gastrointestinal toxicity with celecoxib vs nonsteroidal anti-inflammatory drugs for osteoarthritis and rheumatoid arthritis: the CLASS study: a randomized controlled trial. Celecoxib Long-term Arthritis Safety Study. FAMA 2000;284(10):1247-55.

10. Hrachovec JB, Mora M. Reporting of 6-month vs 12 -month data in a clinical trial of celecoxib [letter]. 7AMA 2001;286:2398.

11. Wright JM, Perry TL, Bassett KL, Chambers GK. Reporting of 6-month vs 12-month data in a clinical trial of celecoxib [letter]. 7AMA 2001;286:2398-400.

12. Melander H, Ahlqvist-Rastad J, Meijer G, Beermann B. Evidence b(i)ased medicine: selective reporting from studies sponsored by pharmaceutical industry: review of studies in new drug applications. BMF 2003;326:1171-3.

13. Kirsch I, Moore TJ, Scoboria A, Nicholls SS. The emperor's new drugs: an analysis of antidepressant medication data submitted to the US Food and Drug Administration. Prevention \& Treatment 2002; 5(Article 23).

14. Writing Group of the Women's Health Initiative. Risks and benefits of estrogen plus progestin in healthy postmenopausal women. 7AMA 2002;288:942-5.

15. Hemminki E, McPherson K. Impact of postmenopausal hormone therapy on heart disease and cancer: pooled data from clinical trials. BM7 1997;315:149-53.

16. Hemminki E, McPherson K. Value of drug-licensing documents in studying the effect of postmenopausal hormone therapy on cardiovascular disease. Lancet 2000;355:566-9.

17. McPherson K, Hemminki E. Synthesising licensing data to assess drug safety. BM7 2004;328:518-20.

Correspondence to: Dr. Joel Lexchin, 121 Walmer Rd., Toronto ON M5R 2X8; fax 416 923-9515; joel.lexchin@utoronto.ca 